\title{
ON EXTENDING SOLUTIONS TO WAVE EQUATIONS ACROSS GLANCING BOUNDARIES
}

\author{
MARK WILLIAMS
}

Introduction. The purpose of this note is to announce some results on the following extension problem. On a $C^{\infty}$ manifold $M$ with boundary, if $u$ is a given extendible distribution satisfying

$$
P u \in C^{\infty}(M),
$$

under what conditions (on $P, \partial M$, and $\left.u\right|_{\partial M}$ ) can $u$ be extended across $\partial M$ as a solution, that is, to a distribution $\tilde{u} \in D^{\prime}(\tilde{M})$ such that $P \tilde{u} \in C^{\infty}(\tilde{M})$, for some open manifold $\tilde{M}$ extending $M$ across $\partial M$ ? Here $P$ is assumed to be a second-order differential operator on $M$ with smooth coefficients, noncharacteristic with respect to $\partial M$, and with real principal symbol $p$ having fiber-simple characteristics

$$
d_{\text {fiber }} p \neq 0 \quad \text { on } \quad p^{-1}(0) \cap\left(T^{*} M \backslash 0\right)
$$

(for example, the wave operator acting in the exterior of a smooth obstacle). After extending the coefficients of $P$ smoothly across $\partial M$, we can view $P$ as an operator on some open extension $\tilde{M}$ of $M$.

The problem is easily solved in the two cases where no null bicharacteristics tangent to $\partial T^{*} M$ are present. When $\partial M$ is everywhere elliptic with respect to $P$, classical theory implies that the desired $\tilde{u}$ can be found if and only if $\left.u\right|_{\partial M} \in C^{\infty}(\partial M)$. When $\partial M$ is everywhere hyperbolic, nothing has to be assumed about $\left.u\right|_{\partial M} \in D^{\prime}(\partial M)$, for the extension $\tilde{u}$ can be produced simply by solving the Cauchy problem in a neighborhood of $\partial M$ with Cauchy data given by $u$. Here we are interested in the two cases where null bicharacteristics tangent to $\partial T^{*} M$ to first order are present, the diffractive and gliding cases. An example given in [8] shows that if the boundary is diffractive, even when $\left.u\right|_{\partial M}$ is smooth, it may happen that no extension as a solution (in fact, no extension $\tilde{u}$ such that $\rho \notin W F P \tilde{u}$ where $\rho \in \partial T^{*} M$ is a point of null bicharacteristic tangency) exists. Our main result (Theorem 2) implies that, in contrast to the diffractive case, near gliding points extensions as microlocal solutions always exist when $\left.u\right|_{\partial M}$ is smooth. We construct such an extension after showing that, near a gliding point $\left.\sigma \notin W F u\right|_{\partial M}$, any distribution $u$ satisfying $P u \in C^{\infty}(M)$ has the series expansion given in Theorem 1. The proof of Theorem 2 makes essential use of the recent unified treatment of the diffractive and gliding parametrices [5], in which the eikonal and transport equations are solved on both sides of the boundary. Full proofs will appear in $[9]$.

We proceed to recall some terminology.

Received by the editors June 21, 1985.

1980 Mathematics Subject Classification (1985 Revision). Primary 35L20.

(c)1986 American Mathematical Society $0273-0979 / 86 \$ 1.00+\$ .25$ per page 
Boundary points. Let $\iota: \partial M \rightarrow M$ be the inclusion inducing the projection $\iota^{*}: \partial T^{*} M \rightarrow T^{*} \partial M$. Then the elliptic, hyperbolic, and glancing regions are respectively

$$
\begin{aligned}
& E=\left\{\sigma \in T^{*} \partial M \backslash 0: p \neq 0 \text { on the line } \iota^{*^{-1}}(\sigma)\right\} . \\
& H=\left\{\sigma \in T^{*} \partial M \backslash 0: p \text { has (two) simple zeros in } \iota^{*^{-1}}(\sigma)\right\} . \\
& G=\left\{\sigma \in T^{*} \partial M \backslash 0: p \text { has a double zero, } \rho, \text { in } \iota^{*^{-1}}(\sigma)\right\} .
\end{aligned}
$$

Letting $x$ be a real $C^{\infty}$ function vanishing simply on $\partial M$ and positive in $\stackrel{\circ}{M}$ near $\partial M$, we write $G=G_{d} \cup G_{g} \cup G_{0}$ (a union of the diffractive, gliding, and higher-order tangency regions), where $\sigma \in G_{d}, G_{g}$, or $G_{0}$ depending on whether $\{p,\{p, x\}\}(\rho)>0,<0$, or $=0$ respectively.

Gliding parametrices. Let $\sigma=\iota^{*}(\rho) \in G_{g}$. Choose coordinates $(x, y, \xi, \eta) \in T^{*} \mathbf{R}^{n+1}$ such that $\stackrel{\circ}{M}=\{x>0\}, \rho=(0,0,0, \bar{\eta})$, and $H_{p} y_{1}(\rho)>$ 0 (so $y_{1}$ will serve as our time variable). The forward and backward gliding parametrices at $\sigma$ are maps $B_{ \pm}: D^{\prime}(\partial M) \rightarrow D^{\prime}(M)$ such that for some small neighborhood $U \subset M$ of $\pi \sigma$ and some small conic neighborhood $\Lambda \subset T^{*} \partial M \backslash 0$ of $\sigma$, we have $P B_{ \pm} g \in C^{\infty}(U)$ and $\left.B_{ \pm} g\right|_{\partial M}=g \bmod C^{\infty}(\partial M)$ for all $g$ with $W F g \subset \Lambda$. Moreover, $B_{+}$(resp. $B_{-}$) propagates singularities in the direction of increasing (resp. decreasing) $y_{1} \cdot B_{ \pm}$(see $[\mathbf{2}, \mathbf{5}$, or $\mathbf{7}]$ ) are constructed from Fourier-Airy integral operators

$$
C_{ \pm} F(x, y)=(2 \pi)^{-n} \int_{\Gamma_{ \pm}} e^{i \phi(x, y, \mu)} I(a, b, \zeta ; x, y, \mu) \hat{F}(\mu) d \mu
$$

where

$$
I=\left[a(x, y, \mu) \operatorname{Ai}(\varsigma(x, y, \mu))+b(x, y, \mu) \operatorname{Ai}^{\prime}(\varsigma(x, y, \mu))\right] / \operatorname{Ai}\left(\zeta_{0}(\mu)\right) .
$$

Here $\mu=\left(z, \mu^{\prime}\right)=\left(z, \mu_{2}, \ldots, \mu_{n}\right) \in \mathbf{C} \times \mathbf{R}^{n-1}$ and $\operatorname{Im} z=\mp T(T>0)$ on $\Gamma_{ \pm}$respectively. Ai is the standard Airy function (see [6, p. 218]), an entire function whose zeros are all simple and negative. $\varsigma_{0}(\mu)=\varsigma(0, y, \mu)=z \mu_{n}^{-1 / 3}$, so $\left(\operatorname{Ai}\left(\varsigma_{0}\right)\right)^{-1}$ makes sense on $\Gamma_{ \pm}$. The phase functions $\phi, \zeta$ and the symbols $a, b$ are obtained by taking almost analytic extensions (see [3]) in the $\mu_{1}$ variable of functions solving eikonal and transport equations on both sides of $x=0$. The symbols $a$ and $b$ are supported in a small conic neighborhood $\nu \subset\left\{(x, y, \mu): \mu_{n} \geq C|\mu|\right\}$ of $(0,0, \bar{\mu})$, where $\bar{\mu}=(0, \ldots, 0,1)$. Finally, we recall that $\phi$ and $b$ also satisfy $\phi_{x, y}^{\prime}(0,0, \bar{\mu})=(0, \bar{\eta})$ and $\left.b\right|_{x=0}=0$.

Operators $B_{ \pm}$with the desired properties can now be obtained by setting $B_{ \pm}=C_{ \pm} J$, where $J: E^{\prime}(\partial M) \rightarrow E^{\prime}\left(\mathbf{R}^{n}\right)$ is a proper elliptic F.I.O. microlocally inverting the boundary operators $\left.\left(C_{ \pm}\right)\right|_{x=0}$.

Note that although $\phi, \zeta, a$, and $b$ are defined on both sides of $x=0$, the operators $B_{ \pm}$are defined only in $x \geq 0$ because the Airy quotients in (3) blow up exponentially in $x<0$.

Main results. We number the zeros $r_{k}$ of $\operatorname{Ai}(z)$ so that $0>r_{1}>r_{2}>$ $\cdots \rightarrow-\infty$. 
THEOREM 1. Let $P$ be a second-order differential operator on $M$ noncharacteristic with respect to $\partial M$, with real principal symbol $p$ satisfying (2). If $\sigma \in G_{g}$ and $u \in D^{\prime}(M)$ satisfies $P u \in C^{\infty}(M)$ and $\left.\sigma \notin W F u\right|_{\partial M}$, then $u=v_{1}+v_{2}$, where $\sigma \notin W F_{b} v_{2}\left(W F_{b}\right.$ is defined in [4]) and $v_{1}=\sum_{k} u_{k}=$ $i(2 \pi)^{1-n} \sum_{k} \int e^{i \phi\left(x, y, \tilde{\mu}_{k}\right)} \alpha_{k} \mu_{n}^{1 / 3}\left[\left(a \operatorname{Ai}(\zeta)+b \operatorname{Ai}^{\prime}(\zeta)\right)\left(x, y, \tilde{\mu}_{k}\right)\right] \hat{F}\left(\tilde{\mu}_{k}\right) d \mu_{2} \cdots d \mu_{n}$. Here $\tilde{\mu}_{k}=\left(r_{k} \mu_{n}^{1 / 3}, \mu_{2}, \ldots, \mu_{n}\right), \alpha_{k}$ is the residue of $(\operatorname{Ai}(z))^{-1}$ at $r_{k}, a, b, \phi, \varsigma$ are as in (3), and $F \in E^{\prime}\left(\mathbf{R}^{n}\right)$.

Since $b(0, y, \mu)=0$ and $\zeta(0, y, \mu)=z \mu_{n}^{-1 / 3}$, each of the terms $u_{k}$ satisfies $\left.u_{k}\right|_{x=0}=0$ as well as $P u_{k} \in C^{\infty}(U)$ for some neighborhood $U \subset M$ of $\pi \sigma$. The factors $\left(\operatorname{Ai}\left(\varsigma_{0}\right)\right)^{-1}$ which forced us to consider only $x \geq 0$ when defining $B_{ \pm}$have now disappeared, so the fact that $\phi, \zeta, a$, and $b\left(x, y, \tilde{\mu}_{k}\right)$ satisfy eikonal and transport equations in a two-sided neighborhood $\tilde{U} \subset \tilde{M}$ of $\pi \sigma$ can be put to use. We deduce that each $u_{k}$ extends to a $\tilde{u}_{k} \in D^{\prime}(\tilde{U})$ such that $P \tilde{u}_{k} \in C^{\infty}(\tilde{U})$. This suggests

THEOREM 2. Let $P$ and $M$ be as in Theorem 1. If $\sigma \in G_{g}$ and if $u \in$ $D^{\prime}(M)$ satisfies $P u \in C^{\infty}(M)$ and $\left.\sigma \notin W F u\right|_{\partial M}$, then an extension $\tilde{u}$ can be constructed such that $W F P \tilde{u} \cap \iota^{*^{-1}}(\Gamma)=\varnothing$, for some conic neighborhood $\Gamma \subset T^{*} \partial M \backslash 0$ of $\sigma$.

Sketch of the proofs. Using the fact that regularity propagates in the boundary near gliding points (see [1]), we first find a distribution $g \in E^{\prime}(\partial M)$, supported in $y_{1}<0$, for which $u=v_{1}+v_{2}$ where $v_{1}=B_{+} g-B_{-} g$ and $\sigma \notin W F_{b} v_{2}$. Putting $F=J g$ we have $(2 \pi)^{n}\left(B_{+} g-B_{-} g\right)=$

$$
\int_{\Gamma_{+}} e^{i \phi} I(a, b, \zeta) \hat{F}\left(z, \mu^{\prime}\right) d z d \mu^{\prime}-\int_{\Gamma_{-}} e^{i \phi} I \hat{F} d z d \mu^{\prime} .
$$

We compute the integral with respect to $z$ first (noting that the integrand has poles at $\left.r_{k} \mu_{n}^{1 / 3}, k=1,2, \ldots\right)$ by taking the limit as $k \rightarrow \infty$ of integrals around closed rectangular contours in $\mathbf{C}$ centered at the origin. In [5] it is shown that the zeros $\sigma_{k}$ of $\operatorname{Ai}^{\prime}(z)$ satisfy $0>\sigma_{1}>r_{1}>\sigma_{2}>r_{2} \cdots \rightarrow-\infty$. So it is convenient to make the left vertical side of the $k$ th rectangle pass through $\sigma_{k+1} \mu_{n}^{1 / 3}$. Estimates of the Airy quotients on the vertical segments show that the contributions to the contour integrals from those segments approach zero as $k \rightarrow \infty$. Thus Cauchy's integral formula with remainder yields that (4) equals

$$
\begin{aligned}
& 2 \pi i \sum_{k} \int e^{i \phi\left(x, y, \tilde{\mu}_{k}\right)} \alpha_{k} \mu_{n}^{1 / 3}\left[\left(a \operatorname{Ai}(\zeta)+b \operatorname{Ai}^{\prime}(\zeta)\right)\left(x, y, \tilde{\mu}_{k}\right)\right] \hat{F}\left(\tilde{\mu}_{k}\right) d \mu^{\prime} \\
& \quad+\iint_{W} \partial_{\bar{z}}\left(e^{i \phi} I(a, b, \zeta) \hat{F}\right) d \bar{z} \wedge d z d \mu^{\prime}
\end{aligned}
$$

where $W=\{z \in \mathbf{C}:-T \leq \operatorname{Im} z \leq T\}$. Though the integrand in the second term has infinitely many poles in $\bar{W}$, estimates of $\left(\operatorname{Ai}\left(\varsigma_{0}\right)\right)^{-1}$ near the negative 
real axis and the fact that $\phi, \zeta, a$, and $b$ are almost analytic in $z$ imply that the second term is smooth in $x \geq 0$. This finishes the proof of Theorem 1 .

As noted above, each $u_{k}$ extends to a $\tilde{u}_{k} \in D^{\prime}(\tilde{U})$ such that $P \tilde{u}_{k} \in C^{\infty}(\tilde{U})$. Let $\chi(y) \in C_{0}^{\infty}\left(\mathbf{R}^{n}\right)$. Then repeated integrations by parts with respect to the $y$ variable yield, for all $N>0$, the estimates $\left|\left\langle\tilde{u}_{k}(x, \cdot), \chi(\cdot)\right\rangle\right| \leq C_{N}\left|\alpha_{k}\right|\left|r_{k}\right|^{-N}$ with $C_{N}$ independent of $k$. Similar estimates clearly hold for $\partial_{x}^{\beta} \tilde{u}_{k}$ as well. Since $r_{k} \sim-c k^{2 / 3}$ (see [5, Appendix A]) and since the residues $\alpha_{k}$ can be shown to satisfy $\left|\alpha_{k}\right| \leq C\left|r_{k}\right|^{-1 / 4}$, we may conclude that the sum $\sum_{k} \tilde{u}_{k}$ converges to a distribution $\tilde{v}_{1} \in C^{\infty}\left(R: D^{\prime}\left(\mathbf{R}^{n}\right)\right)$ extending $v_{1}$. Moreover, for all $N>0,\left|P \tilde{u}_{k}\right| \leq C_{N}\left|r_{k}\right|^{-N}$ in $\tilde{U}$ (by the properties of $\phi, \zeta, a$, and $b$ ), and similar estimates hold for $\partial_{x, y}^{\beta}\left(P \tilde{u}_{k}\right)$. Hence $P \tilde{v}_{1}=\sum_{k} P \tilde{u}_{k} \in C^{\infty}(\tilde{U})$. To complete the proof of Theorem 2 , we invoke a simple lemma $[8,1.6]$ which implies that since $\sigma \notin W F_{b} v_{2}$, an extension $\tilde{v}_{2}$ of $v_{2}$ can be constructed such that $W F \tilde{v}_{2} \cap \iota^{*^{-1}}(\Gamma)=\varnothing$ for some conic neighborhood $\Gamma \ni \sigma$. So $\tilde{u}=\tilde{v}_{1}+\tilde{v}_{2}$ is the desired extension of $u$.

\section{REFERENCES}

1. K. G. Andersson and R. B. Melrose, The propagation of singularities along gliding rays, Invent. Math. 41 (1977), 197-232.

2. G. Eskin, Parametrix and propagation of singularities for the interior mixed hyperbolic problem, J. Analyse Math. 32 (1977), 17-62.

3. A. Melin and J. Sjöstrand, Fourier integral operators with complex valued phase functions, Lecture Notes in Math., vol. 459, Springer-Verlag, Berlin and New York, 1975, pp. 120-223.

4. R. B. Melrose and J. Sjöstrand, Singularities of boundary value problems. I, Comm. Pure Appl. Math. 31 (1978), 593-617.

5. R. B. Melrose and M. Taylor, Boundary problems for wave equations with grazing and gliding rays, monograph (in preparation). 1981 .

6. M. Taylor, Pseudodifferential operators, Princeton Univ. Press, Princeton, N.J.,

7. __ Airy operator calculus, Microlocal Analysis, Contemporary Math., vol. 27, Amer. Math. Soc., 1984, pp. 169-192.

8. M. Williams, Wavefront sets of extensions of solutions to diffractive boundary problems, Comm. Partial Differential Equations 8 (1983), 875-928.

9. __ On extending solutions to Dirichlet problems across the boundary as solutions, Duke Math. J. 52 (1985), 547-563.

\section{Department OF MATHEMATICS, HaRVARD University, CAMbridge, MASSACHUSETTS 02138}

Current address: Department of Mathematics, University of North Carolina, Chapel Hill, North Carolina 27514 